\title{
Effect of Orally Administered Reduced- and Oxidized-Glutathione against Acetaminophen-Induced Liver Injury in Rats
}

\author{
Yoichiro Sugimura and Kazuhiro Yamamoto \\ Tsukuba Research Laboratories, Kyowa Hakko Kogyo, \\ Ibaraki 305-0841, Japan
}

(Received March 23, 1998)

\begin{abstract}
Summary To investigate the effects of orally administered reducedglutathione (GSH) and oxidized-glutathione (GSSG) on liver GSH repletion and liver injury, acetaminophen (AAP) highly loaded rats were used. Orally administered GSH, dependent on dosage, indicated a protective effect against AAP-induced hepatotoxicity. This effect was associated with a recovery of liver GSH levels. Orally administered GSSG also indicated liver GSH recovery and hepatotoxicity inhibition to the same extent as orally administered GSH. Because intraperitoneally administered GSH did not indicate liver GSH recovery, the replenishment of GSH levels after orally administered GSH is thought to be produced through the degradation of GSH into its constituent amino acids in the intestine and their resynthesis in the liver. On the other hand, orally administered GSH indicated a lower liver GSH recovery than orally administered cysteine prodrugs did, although the hepatotoxicity inhibitory degree was similar. Thus orally administered GSH may have another hepatoprotective system besides the resynthesis of its constituent amino acids. The current study establishes that orally administered glutathione, both GSH and GSSG, is a useful tool to recover liver GSH levels and to prevent liver injury.
\end{abstract}

Key Words reduced-glutathione, oxidized-glutathione, acetaminophen, hepatotoxicity, hepatoprotective

Glutathione is a naturally occurring substance that is abundant in many living creatures. It is widely known that a deficiency of reduced-glutathione (GSH) within living organisms can lead to tissue disorder and injury (1). For example, liver injury induced by consuming alcohol (2) or by taking drugs like acetaminophen (3), lung injury by smoking (4), and muscle injury by intense physical activities (5) are all known to be correlated with low tissue levels of GSH. From this point of view,

Abbreviations: GSH, reduced-glutathione; GSSG, oxidized-glutathione; AAP, acetaminophen; DTNB, 5,5'-dithiobis 2-nitrobenzoic acid; MET, methionine; GSH ester, GSH monoethyl ester; OTC, 2-oxothiazolidine-4-carboxylic acid; ALT, L-alanine:2-oxoglutarate aminotransferase; AST, L-aspartate:2-oxoglutarate aminotransferase. 
exogenous GSH supplementation might provide a means to recover reduced GSH levels and to prevent tissue disorders and injuries.

However, the idea of supplying GSH orally is still a conflicting issue (6), mainly for two reasons. The first is the wide belief that GSH is rarely absorbed intact into cells (7). Therefore not GSH, but esters of GSH (8), or cysteine prodrugs (9), have been mainly studied to replete cellular GSH levels. The second reason is that because orally administered GSH is hydrolyzed into its constitutive amino acids in the intestine, GSH itself has had little attention as a means of repleting cellular GSH levels (10). Although some studies have shown the effectiveness of oral GSH against tissue injuries (11-13), only limited numbers of studies have paid attention to its repletion of tissue GSH levels $(1,14)$.

Because orally administered GSH is hydrolyzed into its constituent amino acids in the intestine, these amino acids can be considered beneficial suppliers to replete tissue GSH levels. Thus orally administered GSH itself may be a useful tissue GSH replenisher. Moreover, oxidized-glutathione (GSSG), which is composed of 2 molecules of GSH but has been given little attention for its nutritional effects (15), may also be considered as a tissue GSH replenisher. Moreover, orally administered GSH and GSSG may have some advantages over orally administered cysteine prodrugs. First, GSH and GSSG can be considered as safe tissue GSH repleters, overcoming cysteine prodrugs, which are known to have some toxic effects $(16,17)$. Second, some part of orally administered GSH, but not cysteine prodrugs, may supply GSH directly into the circulation as Hagen et al (18) have reported.

In this paper, we have clearly demonstrated the effectiveness of orally administered GSH and GSSG to replete tissue GSH levels by using acetaminophen (AAP) highly loaded rats, which are widely known models for both hepatic GSH depletion and injury (3). Furthermore, this is the first study that has compared the effectiveness of GSH and GSSG with GSH ester and cysteine prodrugs by using the same hepatotoxicity model by two different administration routes, that is, orally and intraperitoneally.

\section{MATERIALS AND METHODS}

Chemicals. AAP and 5,5'-dithiobis 2-nitrobenzoic acid (DTNB) were purchased from Wako Pure Chemical Industries (Tokyo, Japan). GSH, GSSG, methionine (MET), and GSH monoethyl ester (GSH ester) were supplied by Kyowa Hakko Kogyo (Tokyo, Japan). 2-Oxothiazolidine-4-Carboxylic acid (OTC) was purchased from Sigma Chemical (MO, USA).

Animals. Male Sprague-Dawley rats, supplied by SLC (Hamamatsu, Japan), were kept for a stabilization period of 1 week at $22 \pm 1^{\circ} \mathrm{C}$ and fed a commercial diet (CE-2; Nihon Clea, Tokyo, Japan) and water ad libitum. Light and dark alternated at 12 -h intervals. The body weights of the animals used were from $150 \mathrm{~g}$ to $250 \mathrm{~g}$. 
Preparation of the test solution. AAP was dissolved in $0.9 \%$ saline solution at $80^{\circ} \mathrm{C}$ to give a solution concentration of $35 \mathrm{mg} / \mathrm{mL}$. The following test solutions were dissolved in $0.9 \%$ saline solution to the concentrations described in parentheses: GSH and GSSG $(50.0 \mathrm{mg} / \mathrm{mL}$ or $100.0 \mathrm{mg} / \mathrm{mL})$, OTC $(24.0 \mathrm{mg} / \mathrm{mL})$, Met $(24.0 \mathrm{mg} / \mathrm{mL})$, and GSH ester $(82.7 \mathrm{mg} / \mathrm{mL})$.

Experimental procedure. Rats were fasted for $18 \mathrm{~h}$ before the experiment. They received $0.33-3.26 \mathrm{mmol} / \mathrm{kg}$ of the test solutions orally, and within $1 \mathrm{~h}$ AAP was administered intraperitoneally at a dose of $700 \mathrm{mg} / \mathrm{kg}$. After AAP administration, the rats were anesthesized by the use of pentobarbital sodium $(50 \mathrm{mg} / \mathrm{kg}$; Abbott Laboratories, North Chicago, USA), and blood (from the vena cava) and liver were obtained for further analysis. The blood samples were then centrifuged at $5,000 \times g$ for $15 \mathrm{~min}$ at $4^{\circ} \mathrm{C}$ to obtain serum samples, then stored at $-80^{\circ} \mathrm{C}$. The livers were rinsed in saline solution, then also stored at $-80^{\circ} \mathrm{C}$.

Analytical procedures

Hepatic GSH level: Liver samples of $0.5 \mathrm{~g}$ were homogenized with four volumes of phosphate buffer solution ( $\mathrm{pH} 7.4$ ). An equal volume of $8 \%$ sulfosalytic acid $(\mathrm{w} / \mathrm{v})$ was added to each liver homogenate precipitation. The supernatant was collected by centrifugation at $5,000 \times g$ for $10 \mathrm{~min}$ at $4^{\circ} \mathrm{C}$. The supernatant was used to assay the GSH level by using a slightly modified method described by Reeve et al (19). The modified method is described as follows: $0.5 \mathrm{~mL}$ of supernatant was added with $50 \mu \mathrm{L}$ of $200 \mathrm{~mm}$ DTNB and kept at room temperature for $20 \mathrm{~min} ; 40 \mu \mathrm{L}$ of formic acid was then added and the solution thoroughly mixed. After removing the hydrophobic portion of the supernatant with ethyl acetate, the GSH derivatives in the hydrophilic portions were separated on an STR ODS-M column $(25 \mathrm{~cm} \times$ $4.0 \mathrm{~mm} ; 5 \mu \mathrm{m}$; Shimadzu, Tokyo, Japan) by use of a mobile phase of $0.025 \mathrm{~mm}$ ammonium formic acid, $\mathrm{pH}$ adjusted to 4 by formic acid, and $10 \%$ methanol. The flow rate was $1.0 \mathrm{~mL} / \mathrm{min}$. The GSH-derivatives were detected at $280 \mathrm{~nm}$ and quantified relative to standards by integration (Shimadzu).

Serum transaminase activity: A determination of serum L-alanine:2-oxoglutarate aminotransferase (ALT) and of serum L-aspartate:2-oxoglutarate aminotransferase (AST) activities was performed enzymatically by using the Wako kit No. 431-30901 (Wako Pure Chemical).

Statistical analysis. Results are expressed as means $\pm \mathrm{SE}$ and were analyzed by Student's $t$-test. $p<0.05$ was considered statistically significant.

\section{RESULTS}

Effect of orally administered GSH against liver GSH levels and liver injury

AAP administration resulted in a significant reduction in the liver GSH level, which at $0 \mathrm{~h}$ was $4.9 \mu \mathrm{mol} / \mathrm{g}$, but $2.5 \mathrm{~h}$ after AAP injection it dropped to $0.4 \mu \mathrm{mol} / \mathrm{g}$. After $5 \mathrm{~h}$, the level of GSH was $0.7 \mu \mathrm{mol} / \mathrm{g}$ (Fig. 1). When orally administered GSH $(3.26 \mathrm{mmol} / \mathrm{kg})$ was given before the AAP, however, the liver GSH level at $2.5 \mathrm{~h}$ was $1.5 \mu \mathrm{mol} / \mathrm{g}$, indicating that orally administered GSH can efficiently reduce the Vol 44, No 5, 1998 


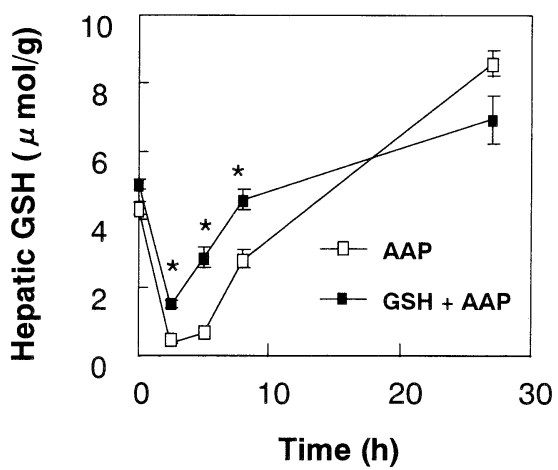

Fig. 1. Effect of orally administered GSH $(3.26 \mathrm{mmol} / \mathrm{kg})$ on hepatic GSH concentrations after AAP $(700 \mathrm{mg} / \mathrm{kg})$ administration. GSH concentrations were determined $0,2.5,5,8$ and $27 \mathrm{~h}$ after administration of GSH. Data are expressed as the mean \pm SE of six rats per time point and group. ${ }^{*}$ denotes significant difference $(p<0.05)$ from the AAP treatment group at that time point.
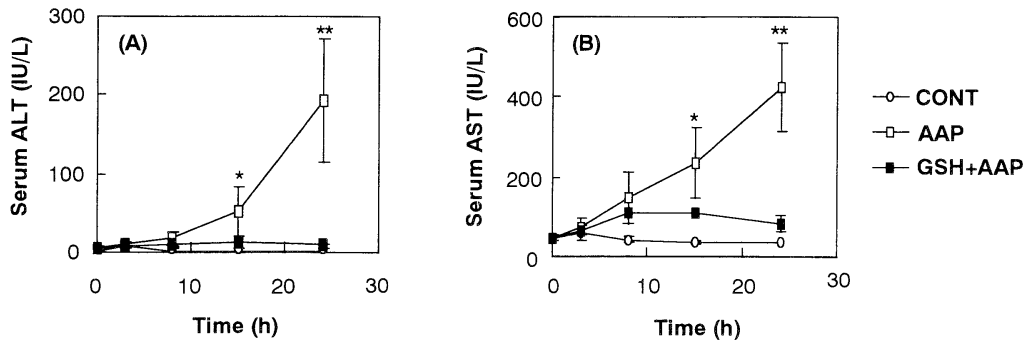

Fig. 2. Effect of orally administered GSH on AAP-induced serum L-alanine:2oxoglutarate aminotransferase (ALT, A) and L-aspartate:2-oxoglutarate aminotransferase activities (AST, B). Enzyme activities were determined 0, 3, 8, 15 and $24 \mathrm{~h}$ after administration of GSH $(3.26 \mathrm{mmol} / \mathrm{kg})$. Data are expressed as means $\pm \mathrm{SE}$ of six rats per time point and group. ${ }^{*}$ and $* *$ denotes significant difference $(p<0.05$ and $p<0.001)$ from values obtained when AAP was administered alone. CONT represents the group without AAP treatment.

fall of liver GSH after AAP injection. Furthermore, the lowered liver GSH level recuperated to original levels more rapidly with orally administered GSH.

Serum ATL and AST were used for liver injury indices. After AAP injection, significant increases of ALT and AST were observed after $15 \mathrm{~h}$ and especially after $24 \mathrm{~h}$ (Fig. 2). Orally administered GSH had the remarkable effect of inhibiting the increase of these serum enzymes.

Dose response of orally administered GSH against liver GSH levels and liver injury

Orally administered GSH, in which its concentrations were $0.33,0.65$, and $1.63 \mathrm{mmol} / \mathrm{kg}$, was given before AAP injection. Five hours after injection, a repletion 


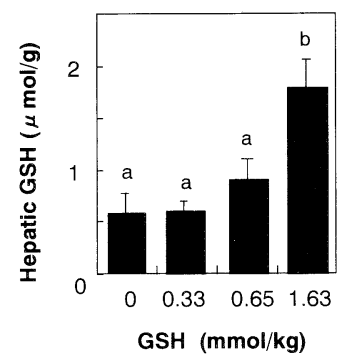

Fig. 3. Dose dependence of orally administered GSH against hepatic GSH levels after AAP $(700 \mathrm{mg} / \mathrm{kg})$ administration. Hepatic GSH concentrations were determined $5 \mathrm{~h}$ after administration of GSH $(0,0.33,0.65$ and $1.63 \mathrm{mmol} / \mathrm{kg})$. Data are expressed as means $\pm \mathrm{SE}$ of three to six rats per time point and group. Data not sharing common subscript letters are significantly different at $p<0.025$.

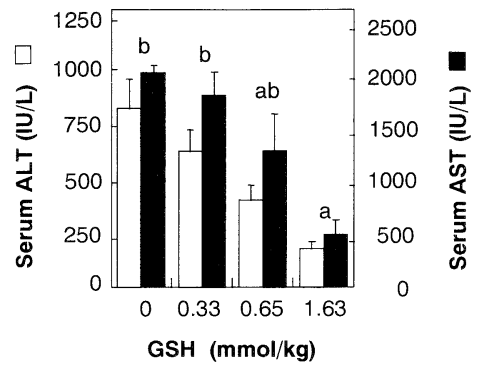

Fig. 4. Dose dependence of orally administered GSH compared against AAP-induced serum L-alanine:2-oxoglutarate aminotransferase (ALT) and L-aspartate:2oxoglutarate aminotransferase activities (AST). Enzyme activities were determined $27 \mathrm{~h}$ after administration of GSH $(0,0.33,0.65$ and $1.63 \mathrm{mmol} / \mathrm{kg})$. Data are expressed as means $\pm \mathrm{SE}$ of three to six rats per time point and group. Data not sharing common subscript letters are significantly different at $p<0.025$.

of the liver GSH levels was shown in a dose response manner. On the other hand, elevation of the serum ALT and AST $27 \mathrm{~h}$ after AAP injection was inhibited in a dose-responsive manner (Figs. 3 and 4).

Comparison of orally administered GSH with orally administered GSSG and other GSH-related compounds

Although GSSG is the oxidized form of GSH, the former has received little attention for its nutritional qualities (15). Thus the effectiveness of orally administered GSSG was compared with that of orally administered GSH in our study.

GSH ester (8) and cysteine prodrugs (9) are known for their abilities to replete tissue GSH levels. Subsequently, the effects of orally administered GSH were also 


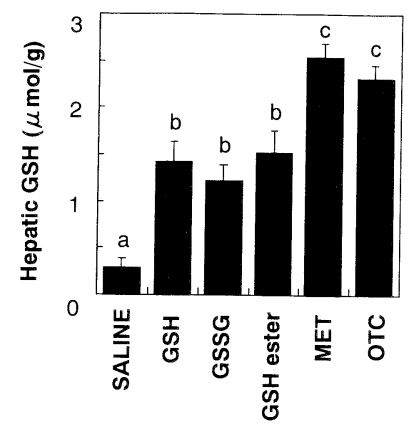

Fig. 5. Effects of orally administered GSH, GSSG, GSH ester, MET and OTC (each administrated $1.63 \mathrm{mmol} / \mathrm{kg}$, except for GSSG; $0.82 \mathrm{mmol} / \mathrm{kg}$ ) on hepatic GSH after AAP $(700 \mathrm{mg} / \mathrm{kg})$ administration. Data are expressed as means \pm SE of six rats per time point and group. Data not sharing common subscript letters are significantly different at $p<0.05$.

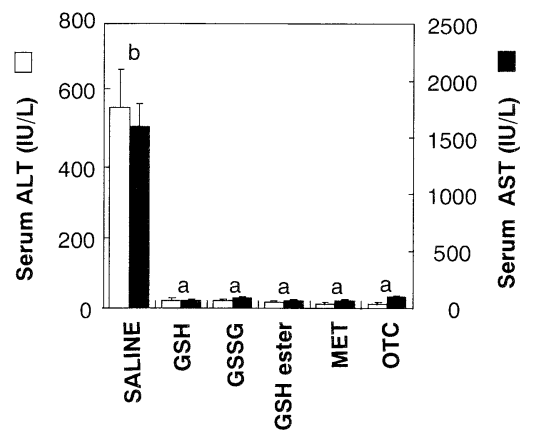

Fig. 6. Effects of orally administered GSH, GSSG, GSH ester, MET and OTC (each administrated $1.63 \mathrm{mmol} / \mathrm{kg}$, except for GSSG $0.82 \mathrm{mmol} / \mathrm{kg}$ ) on AAP-induced serum L-alanine:2-oxoglutarate aminotransferase (ALT) and L-aspartate:2-oxoglutarate aminotransferase activities (AST). Data are expressed as means \pm SE of six rats per time point and group. Data not sharing common subscript letters are significantly different at $p<0.05$.

compared with orally administered GSH ester and cysteine prodrugs (i.e. MET and OTC).

These test solutions were administered $(1.63 \mathrm{mmol} / \mathrm{kg})$ orally $30 \mathrm{~min}$ before AAP injection. GSSG was administered $(0.82 \mathrm{mmol} / \mathrm{kg})$ because it was constituted by 2 molecules of GSH. After AAP injection, the liver GSH level (Fig. 5) and the serum ALT and AST activities (Fig. 6) were determined.

Orally administered GSSG and GSH ester showed liver GSH level recovery and prevented the elevation of serum ALT and AST activities to the same extent as orally administered GSH. Although the cysteine prodrugs (MET and OTC) showed more increased GSH recovery than orally administered GSH, their 

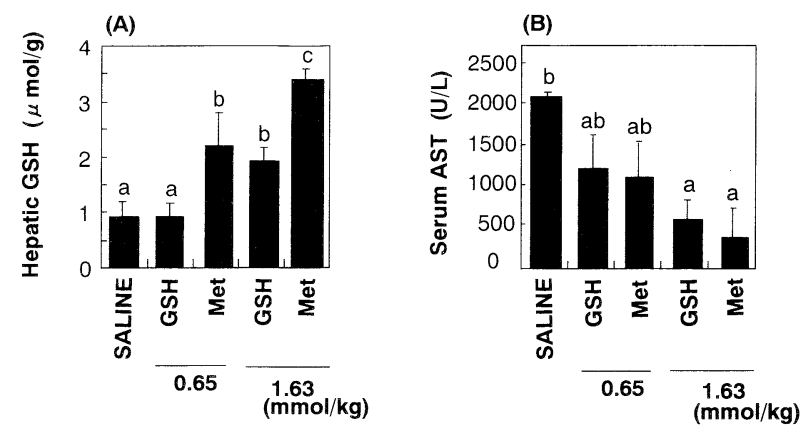

Fig. 7. Dose dependence of orally administered GSH and MET compared to hepatic GSH concentration (A), and to serum L-aspartate:2-oxoglutarate aminotransferase (AST) activities (B). Hepatic GSH concentrations were determined $5 \mathrm{~h}$ and enzyme activities were determined $27 \mathrm{~h}$ after administration of GSH and MET ( 0.65 and $1.63 \mathrm{mmol} / \mathrm{kg}$ ). Data are expressed as means $\pm \mathrm{SE}$ of six rats per time point and group. Data not sharing common subscript letters are significantly different at $p<0.025$.

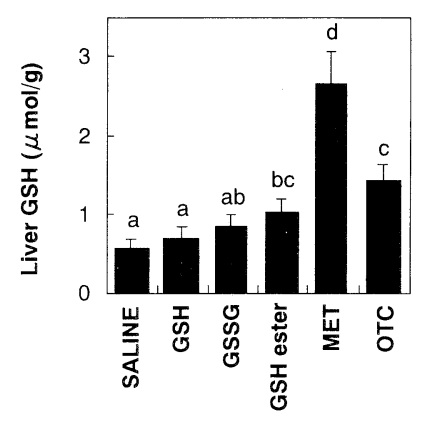

Fig. 8. Effects of intraperitoneally administered GSH, GSSG, GSH ester, MET and OTC (each administrated $1.63 \mathrm{mmol} / \mathrm{kg}$, except for GSSG; $0.82 \mathrm{mmol} / \mathrm{kg}$ ) on hepatic GSH after AAP $(700 \mathrm{mg} / \mathrm{kg})$ administration. Data are expressed as means $\pm \mathrm{SE}$ of six rats per time point and group. Data not sharing common subscript letters are significantly different at $p<0.05$.

prevention of liver injury were the same as that of orally administered GSH. We further compared the dose response of MET to GSH with regard to liver GSH levels and liver injury. Even at a lower dosage $(0.65 \mathrm{mmol} / \mathrm{kg})$, orally administered MET indicated more-efficient liver GSH recovery than orally administered GSH did, and its prevention of liver injury showed the same degree as that of orally administered GSH (Fig. 7).

\section{Effects of intraperitoneally administered GSH and its related compounds}

All test solutions were administered intraperitoneally $(1.63 \mathrm{mmol} / \mathrm{kg}) 30 \mathrm{~min}$ before AAP injection. Because intraperitoneally administered GSH showed maximal 


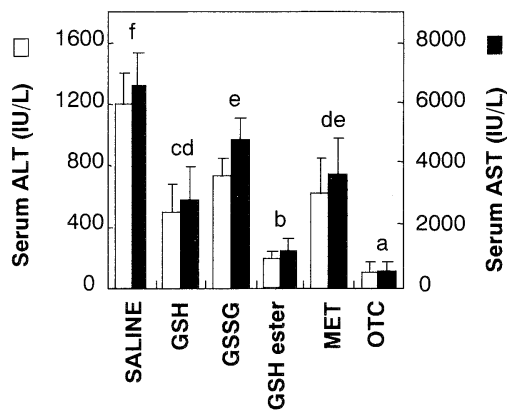

Fig. 9. Effects of intraperitoneally administered GSH, GSSG, GSH ester, MET and OTC (each administrated $1.63 \mathrm{mmol} / \mathrm{kg}$, except for GSSG $0.82 \mathrm{mmol} / \mathrm{kg}$ ) on AAP-induced serum L-alanine:2-oxoglutarate aminotransferase (ALT) and Laspartate:2-oxoglutarate aminotransferase activities (AST). Data are expressed as means \pm SE of six rats per time point and group. Data not sharing common subscript letters are significantly different at $p<0.05$.

plasma GSH concentration within $30 \mathrm{~min}$ (20), GSH-related test solutions also should have been mostly absorbed before AAP administration. Thus interaction between test solutions and AAP should have rarely occurred.

The administration of GSH ester and cysteine prodrugs showed significant liver GSH recovery. However, intraperitoneally administered GSH and GSSG did not indicate GSH repleting effects (Fig. 8).

The GSH ester and OTC prevented increased activities of serum ALT and AST. MET prevented an increase of serum enzyme activities rather weakly despite high levels of liver GSH recovery (Fig. 9). Although GSH and GSSG had no effect on liver GSH repletion, they showed partial prevention against elevated serum ALT and AST activities. In consideration of intraperitoneally administered GSH, the serum enzyme activities surprisingly showed $40 \%$ of the activity compared with the controls used.

\section{DISCUSSION}

AAP is widely used as an antipyretic and analgesic, but its high dosage can result in extensive liver injury associated with lowered liver GSH levels (3). This was also proved in our studies, that fasted rats given AAP $(700 \mathrm{mg} / \mathrm{kg})$ intraperitoneally showed extensive decreased liver GSH levels (Fig. 1) and increased liver injuries (Fig. 2). Consequently, high doses of AAP injected into rats provided a suitable model with which to examine the effects of oral glutathione (both GSH and oxidized-glutathione GSSG).

Orally administered GSH indicated protective effects dose-dependently against AAP-induced hepatotoxicity (Figs. 2 and 4), which was also seen to be associated with a repletion of liver GSH levels (Figs. 1 and 3). Although liver GSH concentration 
is strictly regulated at normal physiological conditions $(21,22)$, our results strongly suggested that orally administered GSH serves as a beneficial replenisher when liver GSH is highly depleted. On the other hand, intraperitoneally administered GSH did not indicate liver GSH repletion (Fig. 8); this effect is because as Meister (6) has reported, liver has no ability to absorb extracellular GSH in an intact form. Furthermore, Vina et al (10) have reported that orally administered GSH cannot replete liver GSH in the case of inhibiting $\gamma$-glutamylcysteine synthase, a rate-limiting enzyme of GSH synthesis. Thus an increase of liver GSH levels by orally administered GSH in our study should have been produced through the degradation of GSH into its constituent amino acids in the intestine by peptidases and $\gamma$-glutamyltranspeptidase, then their de novo resynthesis to GSH in the liver.

Although GSH can be oxidized to GSSG during storage and cooking (23), the nutritional aspects of orally administered GSSG are not well known. In this paper, orally administered GSSG showed liver GSH recovery and liver injury inhibition to the same extent as orally administered GSH (Figs. 5 and 6). Hagen et al (24) reported that GSSG could be reduced to GSH in the gut. Therefore orally administered GSSG might have followed the same fate as orally administered GSH after being reduced to GSH. From results indicated in our study, orally administered GSSG is as beneficial as orally administered GSH for liver GSH recovery and liver injury inhibition.

GSH esters and cysteine prodrugs have been widely used to study tissue GSH repletion. GSH esters, which are more lipophilic than GSH is, can penetrate cells and increase tissue GSH levels (8). Cysteine prodrugs like methionine (MET) (25) or 2-oxothiazolidine-4-carboxylic acid (OTC) (9) are widely used because cysteine is the rate-limiting compound for GSH synthesis. In this paper, the effects of GSH and GSSG were compared with those of GSH esters and cysteine prodrugs using the AAP-induced hepatotoxicity model.

Although intraperitoneally administered GSH esters showed liver GSH repletion (Fig. 8), orally administered GSH ester achieved the same extent of liver GSH recovery as that of GSH (Fig. 5) and showed no particular advantage over GSH repletion. A possible explanation of this effect may be that orally administered GSH ester was hydrolyzed in the gut to its constituents, as Grattagliano et al (26) had mentioned, in the same manner as orally administered GSH was.

Orally administered cysteine prodrugs indicated 1.5 times higher GSH level than orally administered GSH did (Fig. 5). Because Vina et al (10) had reported that orally administered GSH slowly generates cysteine through the combined action of intestinal $\alpha$-dipeptidases and $\gamma$-glutamyl transpeptidase, our result could have indicated that orally administered cysteine prodrugs supplied cysteine faster to the liver than orally administered GSH did. Intraperitoneally administered cysteine prodrugs also prevented liver injury by acting as a GSH precursor (Figs. 8 and 9), which was reported elsewhere (6). Although intraperitoneally administered MET efficiently recovered liver GSH level, its prevention of liver injury was low (Figs. 8 and 9). One reason for this may be that MET entered the circula- 
tion so rapidly that its toxic effect, as Benevenga (16) reported, had exaggerated. Orally administered OTC has shown some side effects in human tests, which were reported by Porta et al (17). In our study, OTC increased the liver malondialdehyde level (unpublished observations). Consequently, cysteine prodrugs may be considered not suitable GSH precursors because of their side effects; thus GSH itself may be the best-suited compound.

Even though orally administered GSH and GSSG indicated a lower liver GSH recovery than orally administered cysteine prodrugs did (Fig. 5), their hepatoprotective extent was similar (Fig. 6). The same result could also be seen when dosage was lowered (Fig. 7), which suggests that this phenomenon cannot be explained by the GSH threshold level. In consideration of these results, orally administered GSH may have another hepatoprotective system besides the resynthesis of its constituent amino acids. Because intraperitoneally administered GSH showed a slight but significant hepatoprotective effect (Fig. 9), it might have played an important role in preventing liver injury from the extracellular site of the liver as Liu et al had reported (27). Furthermore, Hagen et al (18) had reported that orally administered GSH enhanced plasma GSH levels. In consideration of these two studies, the hepatoprotective effect of orally administered GSH mainly depends on its hepatic GSH replenishment, but on the other hand, a small amount of orally administered GSH might have been absorbed intact, and this might have strengthened its hepatoprotective activity.

The current study clearly demonstrates that orally administered glutathione itself, both GSH and GSSG, is a useful tool to replete tissue GSH level. It also showed the same extent of liver GSH recovery and hepatoprotection as GSH ester, which is a well-known tissue GSH replenisher. By comparing the effects of orally administered GSH or GSSG with those of cysteine prodrugs, GSH and GSSG showed the same extent of hepatoprotection as that of cysteine prodrugs, although the latter indicated higher liver GSH recovery. This may indicate that orally administered GSH may be useful beyond GSH repletion and that it may have some alternative hepatoprotective mechanism besides GSH repletion. Our study, clearly indicated orally administered GSH and GSSG as beneficial liver GSH repleters, suggests the possibility of carrying out more studies in other physiological situations in which GSH depletion occurs, for instance, in situations of excessive alcohol consumption, smoking, and exhaustive physical exercises.

\section{REFERENCES}

1) White AC, Thannickal VJ, Fanburg BL. 1994. Glutathione deficiency in human disease. $J$ Nutr Biochem 5: 218-226.

2) Videla LA, Valenzuela A. 1981. Alcohol ingestion, liver glutathione and lipoperoxidation: metabolic interrelations and pathological implications. Life Sci 31: 2395-2407.

3) Mitchell JR, Potter DJ, Potter WZ, Davis DC, Gillette JR, Brodie BB. 1973. 
Acetaminophen-induced liver necrosis. 1. Role of drug metabolism. J Pharmacol Exp Ther 187: 185-194.

4) Rahman I, Li XY, Donaldson K, Harrison DJ, MacNee W. 1995. Glutathione homeostasis in alveolar epithelial cells in vitro and lung in vivo under oxidative stress. Am J Physiol 269: L285-L292.

5) Leeuweburgh C, Ji LL. 1995. Glutathione depletion in rested and exercised mice: biochemical consequence and adaptation. Arch Biochem Biophys 316: 941-949.

6) Meister A. 1984. New aspects of glutathione biochemistry and transport-selective alteration of glutathione metabolism. Nutr Rev 42: 397-419.

7) Jensen GL, Meister A. 1983. Radioprotection of human lymphoid cells by exogenously supplied glutathione is mediated by gamma-glutamyl transpeptidase. Proc Natl Acad Sci USA 80: 4714-4717.

8) Anderson ME, Powrie F, Puri RN, Meister A. 1985. Glutathione monoethyl ester: preparation, uptake by tissues, and conversion to glutathione. Arch Biochem Biophys 239: 538-548.

9) Hazelton GA, Hjelle JJ, Klaassen CD. 1986. Effects of cysteine pro-drugs on acetaminophen-induced hepatotoxicity. J. Pharmacol Exp Ther 237: 341-349.

10) Vina J, Carmen P, Furukawa T, Palacin M, Vina JR. 1989. Effect of oral glutathione on hepatic glutathione levels in rats and mice. Br J Nutr 62: 683-691.

11) Gorla N, de Ferreyra EC, Villarruel MC, Fenos OM, Castro JA. 1983. Studies on the mechanism of glutathione prevention of carbon tetrachloride-induced liver injury. $\mathrm{Br}$ $J$ Exp Pathol 64: 388-395.

12) Karmali RA. 1984. Growth inhibition and prostaglandin metabolism in the R3230AC mammary adenocarcinoma by reduced glutathione. Cancer Biochem Biophys 7: 147-154.

13) Videla LA. 1990. Glutathione and alcohol. In: Glutathione: Metabolism and Physiological Functions (Vina J, ed), p 57-67. CRC Press, Boston.

14) Furukawa T, Meydani SN, Blumberg JB. 1987. Reversal of age-associated decline in immune responsiveness by dietary glutathione supplementation in mice. Mech Ageing Dev 38: 107-117.

15) Neuhauser-Berthold M, Kuhfus A, Bassier KH. 1988. Utilization of glutathione disulfides as cysteine source during long-term parenteral nutrition in the growing rat. Metabolism 37: 796-801.

16) Benevenga NJ. 1974. Toxicities of methionine and other amino acids. J Agric Food Chem 22: 2-9.

17) Porta P, Aebi S, Summer K, Lauterburg BH. 1991. L-2-Oxothiazolidine-4-carboxylic acid, a cysteine prodrug: pharmacokinetics and effects on thiols in plasma and lymphocytes in human. J Pharmacol Exp Ther 257: 331-334.

18) Hagen TM, Wierzbicka GT, Sillau AH, Bowman BB, Jones DP. 1990. Bioavailability of dietary glutathione: effect on plasma concentration. Am J Physiol 259: G524-G529.

19) Reeve J, Kuhlenkamp J, Kaplowitz N. 1980. Estimation of glutathione in rat liver by reversed-phase high-performance liquid chromatography separation from cysteine and gamma-glutamylcysteine. J Chromatogr 194: 424-428.

20) Sen CK, Atalay M, Hanninen O. 1994. Exercise-induced oxidative stress: glutathione supplementation and deficiency. $J$ Appl Physiol 77: 2177-2187.

21) Aw TY, Wierzbicka G, Jones DP. 1991. Oral glutathione increases tissue glutathione in vivo. Chem Biol Interactions 80: 89-97.

22) Bauman PF, Smith TK, Bray TM. 1988. The effect of dietary protein and sulfur amino acids on hepatic glutathione concentration and glutathione-dependent enzyme activities 
in the rat. Can J Physiol Pharmacol 66: 1048-1052.

23) Wierzbicka GT, Hagen TM, Jones DP. 1989. Glutathione in food. J Food Comp Anal 2: $327-337$.

24) Hagen TM, Wierzbicka TGT, Bowman BB, Aw TY, Jones DP. 1990. Fate of dietary glutathione: disposition in the gastrointestinal tract. Am J Physiol 259: G530-G535.

25) Tateishi N, Hirasawa M, Higashi T, Sakamoto Y. 1982. The L-methionine-sparing effect of dietary glutathione in rats. $J$ Nutr 112: 2217-2226.

26) Grattagliano L, Wieland P, Schranz C, Lauterburg BH. 1994. Effect of oral glutathione monoethyl ester and glutathione on circulating and hepatic sulfhydrils in the rat. Pharmacol Toxicol 75: 343-347.

27) Liu P, Fisher MA, Farhood A, Smith CW, Jaeschke H. 1994. Beneficial effects of extracellular glutathione against endotoxin-induced liver injury during ischemina and repurfusion. Circ Shock 43: 64-70. 